\title{
METHOD OF CALCULATING INTERMEDIATE DIAMETRAL SIZES AND ALLOWANCES FOR DESIGNING TECHNOLOGY OF MANUFACTURE OF DETAILS
}

\author{
Khalimonenko A.D. \\ Department of Mechanical Engineering \\ St. Petersburg Mining University \\ Russia \\ khalim76@ rambler.ru
}

\author{
Pompeev K.P. \\ Department of Instrument Technology \\ St. Petersburg National Research University of Information \\ Technologies, Mechanics and Optics \\ Russia \\ kir-pom@mail.ru
}

\author{
Timofeev D.Yu. \\ Department of Mechanical Engineering \\ St. Petersburg Mining University \\ Russia \\ duck-tim63@yandex.ru
}

\begin{abstract}
The method of calculation of intermediate diametrical sizes and allowances is considered in the article when designing the technology of manufacturing machine parts. This method makes it possible to ensure the quality of manufacturing machine parts by preliminarily determining the adjustment dimensions before machining. The proposed method allows determining allowances for machining, taking into account the error of the previous processing.
\end{abstract}

Keywords - calculation of allowances, machining, processing technology of machine parts, quality of machine parts, calculation of beats, eccentricity.

\section{INTRODUCTION}

Analyzing standard ISO 8402-94, which defines the concept of "product quality", it is possible to characterize it as a set of properties and signs of products, goods, services, which determine their ability to meet the needs and demands of people, meet their purpose and requirements. At the same time, quality is determined by the measure of the conformity of goods and services with the conditions and requirements of standards, contracts, contracts, customer requests. In addition, quality is the main tool to reduce costs, that is, it is always cheaper to produce correctly the first time than to eliminate the error later [1].

Technical quality of products is laid and provided at the stages of research, development (design) and production. At the same time, technological support of quality indicators for parts begins already at the design stage $[1,2]$.

At the present stage of development of production, the following point of view on the quality of products is finally established, based on the fact that the buyer must purchase defect-free products. This means that the quality of the product offered to the consumer should not be 90 or $99 \%$, but $100 \%$, and its production must be conducted without a correctable, and even more so, an incorrigible marriage [3].

The quality of products is determined by the system of indicators. Among them, first of all, the product designation indicators that characterize the main functions of the product and the scope of its application, and which include classification, operational and structural indicators, are highlighted. The latter, in particular, include the dimensions (subject to their tolerances) of the product and its parts $[3,4]$.

Ensuring the quality of manufacturing parts has always been and will be an urgent problem, as long as there is an industrial production.

In any production, the quality of the product is closely related to the accuracy of manufacturing parts that make up its composition. It is known that the precision of the part allows understanding whether it meets the requirements of the drawing or not: on the sizes, the geometrical form, correctness of an interposition of processed surfaces and on degree of their roughness [5].

The specified accuracy is obtained (provided) by one of two methods: either by trial passes and measurements, or by automatically obtaining dimensions on pre-tuned equipment [6]. In the conditions of automated production, for example, when using CNC machines, the second method is preferable.

When using the automatic dimensioning method on preconfigured machines, the task of ensuring the required processing accuracy is transferred from the machine tool to the machine adjuster or programmer. The advantages of this method are obvious - this is an increase in the accuracy of processing and a reduction in rejection; increased processing capacity; a reduction of qualification requirements for workers; an increase in the economy of production. 
The technological process (TP) of manufacturing a part is, as a rule, a multi-operation process. Therefore, to efficiently implement the method of automatic sizing on pre-tuned equipment in production, it is necessary to calculate all intermediate (linear and diametric) dimensions and allowances for processing its surfaces beforehand at the design stage of the TP.

With the automated design of routing technological processes (RTP) for the manufacture of parts, especially axisymmetric, it is important to develop such MTFs which, at the design stage, would reliably guarantee the required accuracy of manufacturing parts and specified roughness parameters of the treated surfaces. That is, they would allow abandoning the experimental and installation batches during the introduction of designed TP in production. This is facilitated by appropriate calculation methods.

The establishment of optimal allowances for processing has a significant technical and economic importance in the development of TP parts.

The increased allowances cause material overexpenditure in the manufacture of parts and the need to introduce additional technological transitions, to increase the laboriousness of the processing processes, the consumption of the cutting tool, to increase the prime cost of processing the workpiece.

The reduced allowances do not provide removal of defective surface layers and obtainment of the required accuracy and roughness of the treated surfaces, and in some cases create unacceptable conditions for the cutting tool to work on the casting crust or scale. As a result of insufficient allowances, rejects increase, which leads to an increase in production cost.

Initially, an experimental and statistical method of establishing allowances based on available tables for general and intermediate allowances was widely used in industry. A significant drawback of this method is that the experimental and statistical allowances are in many cases overstated, since they are oriented to processing conditions, in which the allowance should be the largest to avoid a reject. At it appointment, the technologist does not analyse the conditions of performing operations.

In this connection, a calculation and analytical method for determining allowances has arisen. According to this method, the intermediate allowance having the minimum value should be such that when it is removed, various defects and inaccuracies, obtained in the previous technological transitions and arising on the performed transition, are eliminated. This question will be discussed in more detail below. This method of establishing allowances (a generally accepted method), developed by prof. V.M. Kovan, is based on taking into account the specific conditions for performing TP of processing and is described in detail in [7]. When designing the TP for manufacturing axisymmetric parts, the method of professor V.M. Kovan is used both for calculation of interoperational linear dimensions, and for determining intermediate diametric dimensions.

With reference to the determination of intermediate diametrical dimensions by the method of professor V.M. Kovan, there is also an alternative calculation and analytical method, described in [8] and developed in due time in NorthWestern Polytechnic Institute (Saint Petersburg, Russia).

Let us compare these two methods for calculating the intermediate diametrical sizes and allowances in the design of manufacturing techniques for axisymmetric parts and for identifying the significant drawbacks of the first method.

\section{COMPARATIVE ANALYSIS OF GENERAL AND ALTERNATIVE METHODS}

At the heart of both methods, there is the calculation of the minimum allowance $\left(z_{\min i}\right)$.

According to the conventional method, the minimum allowance should be such that, when removing it, the surface layer defects $\left(R z_{i-1}\right.$ и $\left.h_{i-1}\right)$ and the shaping or processing the errors $\left(\Delta_{\Sigma i-1}\right)$, obtained at the previous technological transitions, are eliminated, preforms $\left(\varepsilon_{i}\right)$, appearing on the performed transition [7]. Its value is determined by summing these components. When determining the allowance necessary for the processing of external and internal surfaces, the socalled two-sided (double minimum) allowance is calculated. All the components of the minimum allowance $\left(R z_{i-1}, h_{i-1}, \Delta_{\Sigma i-1}\right.$ и $\varepsilon_{i}$ ) are reference data. In view of the vector nature of the errors $\Delta_{\Sigma i-1}$ and $\varepsilon_{i}$, their vector sum is determined as the square root of the sum of the squares of these errors.

According to the alternative method, the minimum allowance for the side is determined, taking into account surface layer defects $\left(R z_{i-1}\right.$ и $\left.h_{i-1}\right)$ and vector sum $\Delta_{\Sigma i-1}$ and $\varepsilon_{i}$ is replaced by the unevenness of the allowance $\left(e_{\max }\right)$. Unevenness of the allowance is the total vector value of the spatial deviations arising during the considered and previous operations (transitions), and includes the error of installation and uncontrolled errors in the shape or processing. It is determined based on the calculation of the beats, arising during the production and processing of the surfaces of rotation of the workpiece along the entire TP of manufacturing the axisymmetric part [8]. Unevenness of the allowance is determined only after checking the possibility of automatic provisioning without reconciliation (or with reconciliation) of the specified requirements for the mutual location (radial beats or misalignments) of the revolution surfaces between themselves. However, this issue is not considered in this article. Thus, the unevenness of the $e_{\max }$ allowance is the design value, not the reference parameter.

According to the conventional method, taking into account the two-sided allowance, the minimum diametrical size of the previous machining stage $\left(D_{\min i-1}\right)$ for the shaft or the maximum diametrical size of the previous machining stage $\left(D_{\max i-1}\right)$ for the hole are calculated by the following formulas:

$$
\begin{aligned}
& D_{\min i-1}=D_{\min i}+2 z_{\min i} \\
& D_{\max i-1}=D_{\max i}-2 z_{\min i}
\end{aligned}
$$

where $\mathrm{D}_{\min i}$ and $D_{\max i}$ are, respectively, the minimum and maximum diametrical sizes of the processing stage to be performed.

Using formulas (1) and (2) and expressing the minimum allowance through diametrical dimensions, the authors obtain the formulas for calculating the corresponding values for the shaft and the hole: 


$$
\begin{aligned}
& z_{\min i}=\left(D_{\min i-1}-D_{\min i}\right) / 2 \\
& z_{\min i}=\left(\operatorname{Dmax} i-D_{\max i-1}\right) / 2
\end{aligned}
$$

Let us analyze formulas (3) and (4) from the viewpoint of the correspondence of the theory of dimensional chains. These expressions are three-tier dimensional chains in which the closing link is the minimum allowance, and the constituent links are the two diametrical sizes of the previous and performed stages of processing of the same surface of revolution. One of the constituent links is increasing, and the second (with a minus sign) is decreasing. In this case, in accordance with the theory of dimensional chains, when determining the minimum of the closing link, it is necessary to subtract the maximum value of the decreasing link from the minimum value of the increasing link. Then the formulas (3) and (4) must accordingly have the following form:

$$
\begin{aligned}
& \mathrm{z}_{\min i}=\left(D_{\min i-1}-D_{\max i}\right) / 2=z_{\min i}^{\mathrm{f}} \\
& \operatorname{z\operatorname {min}i}=\left(D_{\min i}-D_{\max i-1}\right) / 2=z_{\min i}^{\mathrm{f}}
\end{aligned}
$$

It is these formulas (5) and (6) that are used in the alternative method to determine the actual minimum allowance $\left(z_{\min i}^{\mathrm{f}}\right)$ for machining shafts and holes, respectively.

Thus, formulas (3) and (4) contradict the theory of dimensional chains. This contradiction for the case of shafts (external surfaces of rotation) is clearly illustrated in Figure 1, which shows that there is an allowance of a smaller value than the minimum allowance, calculated by the method of professor V.M. Kovan. Accordingly, the allowance is larger than the maximum allowance calculated by the same method.

Also from this figure, it is seen that the minimum allowance on the side includes half the tolerance for the diametrical size of the performed transition (the processing stage being performed). At the same time, this parameter is not included in the equation for calculating the minimum allowance determined by summing parameters $R z_{i-1}, h_{i-1}, \Delta_{\Sigma i-1}$ и $\varepsilon_{i}$. It is noted that according to the method of professor V.M. Kovan the doubled (two-sided) allowance on the executed transition can't be less than the tolerance for the diametrical size of the resulting surface of rotation.

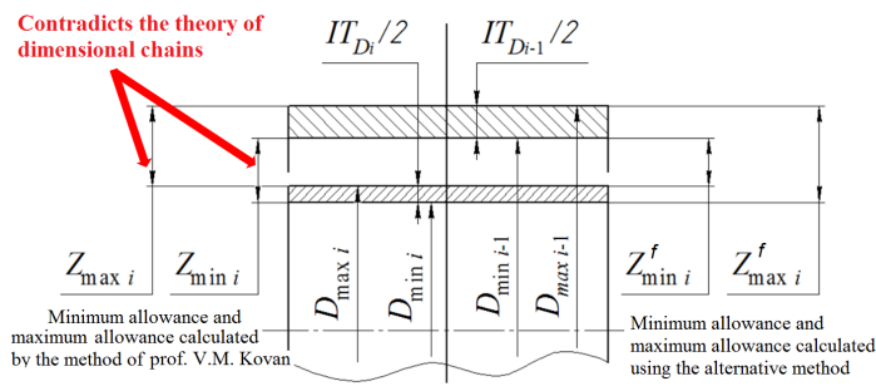

Fig. 1. Graphical representation of allowances for processing the external surface (taking into account its closest states) by the method of professor V.M. Kovan and in accordance with an alternative method for their determination

In [7], an example of a calculation card for processing allowances and size limits for technological transitions for an elementary surface is presented. Let us analyze it from the point of view of convenience of use and simplicity of automation of the calculation process. The map in question and an illustration of some of the results of its analysis are shown in Figure 2.

As can be seen from Fig. 2, calculation of processing allowances and size limits for technological transitions for an elementary surface goes in the direction from its state, which corresponds to the state required in the workpiece (D), in its initial workpiece (3). That is, the direction of calculation does not correspond to the sequence of formation of the data array. This, on the one hand, is inconvenient to use, and on the other hand, complicates the corresponding algorithms and programs.

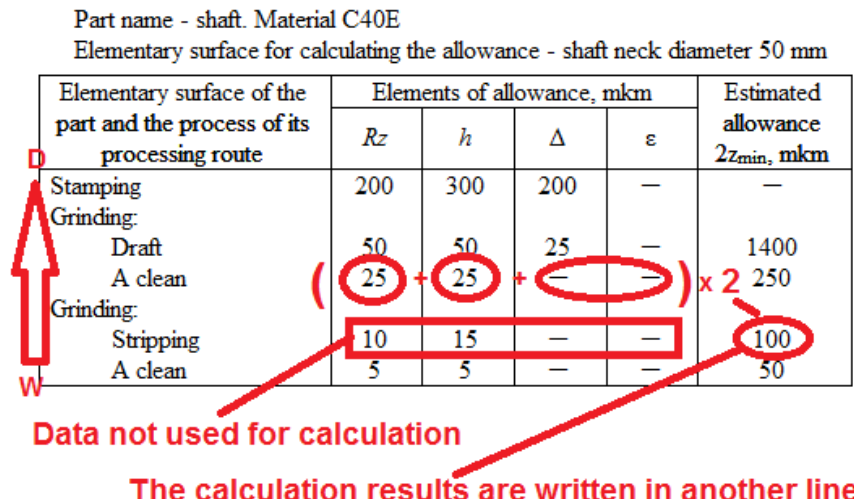

Fig. 2. The calculation card for processing allowances and size limits for technological transitions for an elementary surface

The card contains data that is not used in the calculation.

The result of determining the estimated minimum allowance is written in another line, that is, not in the line in which its components are presented, and their sum must be multiplied by two in the mind. All this makes it difficult to verify the correctness of the calculations performed, and also complicates the computational algorithms and programs.

If we graphically represent the results of calculating the diametric dimensions from the transitions (see Figure 3), we can see the following picture. Cases are possible at finishing and finishing operations or transitions, when the value of the actual minimum allowance, calculated with allowance for the positions of the theory of dimensional chains, turns out to be zero. That is, formally there is no minimum allowance allowing to provide both the cutting process during the surface treatment and the necessary roughness obtained after that. Consequently, there is a probability of replacing the cutting process by plastic deformation of the treated surface, as well as obtaining a correctable or incorrigible reject. The latter is unacceptable from the point of view of reliable quality assurance of the manufacture of axisymmetric parts by accuracy parameters.

Thus, the analysis of the conventional method (Prof. V.M. Kovan's method) revealed the following shortcomings:

1. Calculation of the minimum and standard allowances contradicts the theory of dimensional chains.

2 . If one calculates the values of the minimum allowances based on diametrical dimensions by the method of professor 
Kovan, but with the maximum-minimum method, we can get them equal to zero, hence, there is a probability:

- replacement of the cutting process by plastic deformation of the treated surface;

- receiving a recoverable or permanent reject.

\begin{tabular}{|c|c|c|}
\hline \multirow{2}{*}{$\begin{array}{l}\text { Elementary surface of the } \\
\text { part and the process of its } \\
\text { processing route }\end{array}$} & \multicolumn{2}{|c|}{$\begin{array}{c}\text { Accepted (rounded) } \\
\text { dimensions for transitions, mm }\end{array}$} \\
\hline & $d_{\max }$ & $d_{\min }$ \\
\hline $\begin{array}{l}\text { Stamping } \\
\text { Grinding: }\end{array}$ & 54,00 & 52,00 \\
\hline Draft & 50,90 & 50,40 \\
\hline $\begin{array}{l}\text { A clean } \\
\text { Grinding. }\end{array}$ & 50,25 & 50,10 \\
\hline $\begin{array}{l}\text { Stripping } \\
\text { A clean }\end{array}$ & $\begin{array}{l}50,10 \\
50,00\end{array}$ & $\begin{array}{l}50,00 \\
49,95\end{array}$ \\
\hline
\end{tabular}

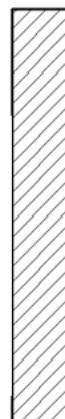

From the position of the theory of dimensional chains

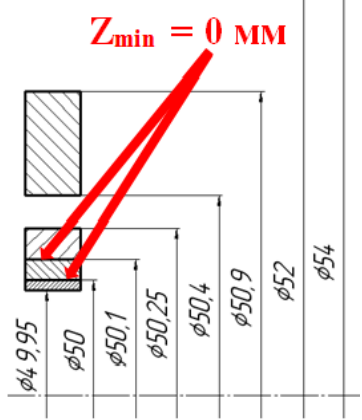

Fig. 3. Graphical representation of the results of calculating the limiting dimensions for technological transitions for an elementary surface by the method of professor V.M. Kovan

3. The probability of obtaining a reject in turn entails the need for the introduction of a designed TP based on the results of pilot and installation batches.

4. Redundancy of information not used in the calculation.

5. The direction of calculation does not correspond to the procedures for creating an array of data, which complicates the development of appropriate algorithms and programs.

6. The result of calculating the doubled minimum allowance is written in another line, not storing the data about the values of the components of this allowance. This also complicates the development of appropriate algorithms and programs.

\section{CALCULATION OF ALLOWANCES BY AN ALTERNATIVE METHOD}

Such drawbacks are deprived of an alternative method of calculating intermediate diametrical sizes and allowances. As it was said above, this method does not completely contradict the theory of dimensional chains since it was developed taking into account its provisions. In addition, it is based on the use of the calculation of diametric dimensions by the maximumminimum method [8], which makes it possible to ensure complete interchangeability, that is, the absence of any rejection in the manufacture of axisymmetric parts. The error in setting the workpiece to be machined is calculated using the empirical formulas presented in [9]. It is best suited for the conditions of automated design of routing technological processes for manufacturing axisymmetric parts.

In Figure 4, as an example, a table is presented with the calculation of intermediate diametric dimensions and allowances for processing the outer cylindrical surface of an axisymmetric part, along with a graphical representation of the results of calculating diametrical dimensions. As can be seen from Fig. 4, the direction of the calculation corresponds to the sequence of formation of the data array from the final state of the surface, corresponding to its state in the part, which diametrical size is already known at the beginning of the calculation, to the state of this surface in the initial workpiece, which diametrical size should be calculated.

\begin{tabular}{|c|c|c|c|c|c|}
\hline \multirow{2}{*}{$\begin{array}{l}\text { Processing } \\
\text { route of the } \\
\text { element }\end{array}$} & \multirow{2}{*}{$\begin{array}{c}\text { № of } \\
\text { operation }\end{array}$} & \multicolumn{2}{|c|}{ Dimension, $\mathrm{mm}$} & \multicolumn{2}{|c|}{ Deviations, mm } \\
\hline & & \begin{tabular}{|l} 
Calculated \\
\end{tabular} & Accepted & & \\
\hline 1 & 2 & 3 & 4 & \multicolumn{2}{|c|}{5} \\
\hline \multicolumn{6}{|l|}{$\varnothing 110-0,35$} \\
\hline $\begin{array}{l}\text { Half-turn } \\
\text { turning }\end{array}$ & 020 & 110 & 110 & \multicolumn{2}{|c|}{$-0,35$} \\
\hline Draft turning & 020 & 111,326 & 111,4 & \multicolumn{2}{|c|}{$-0,87$} \\
\hline Stamping & 005 & 112,96 & 113 & \multicolumn{2}{|c|}{$\begin{array}{l}+1,0 \\
-0,4\end{array}$} \\
\hline \multicolumn{3}{|c|}{ Allowance elements, mm } & \multirow[t]{2}{*}{$\begin{array}{c}\text { Allowance, } \\
\mathrm{mm}\end{array}$} & \multicolumn{2}{|c|}{$\begin{array}{c}\text { Actual allowance, } \\
\mathrm{mm}\end{array}$} \\
\hline$R_{z \mathrm{i}-1}$ & $h_{\mathrm{i}-1}$ & $e_{\max }$ & & $Z_{\min }^{i}$ & $Z_{\max }$ \\
\hline 6 & 7 & 8 & 9 & 10 & 11 \\
\hline 0,1 & $(0,1)$ & 028 & 0,228 & 0,265 & 0,875 \\
\hline 0,16 & 0,2 & 0,22 & 0,58 & 0,6 & 1,735 \\
\hline $\begin{array}{lll}- & & \\
\end{array}$ & $\begin{array}{ll}- \\
-\end{array}$ & - & $\begin{array}{ll}- \\
-\end{array}$ & $\begin{array}{lll}- & & \\
-\end{array}$ & $\begin{array}{ll}- & \\
\end{array}$ \\
\hline
\end{tabular}

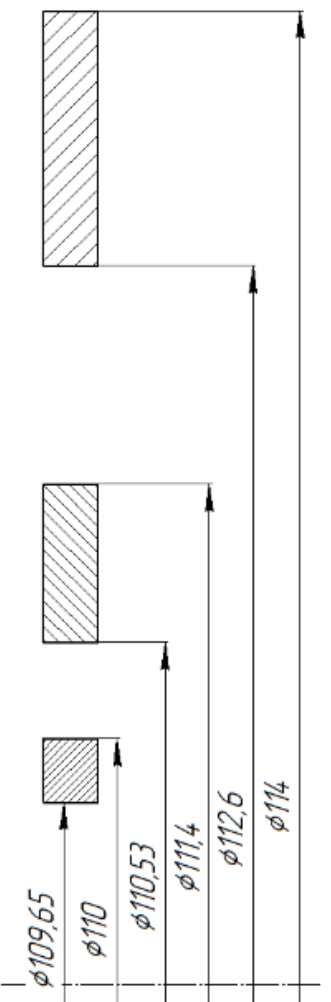

Fig.4. Table with the results of calculating the intermediate diametrical dimensions and allowances for processing the outer cylindrical surface of the axisymmetric part and their graphical representation 
The value of the minimum estimated allowance for the side for the performed transition is written in the same row as the values of its constituent elements are (Fig. 4).

Calculation of intermediate diameters is carried out with the selection in the necessary cases of a "clean" treatment zone to take into account the causes, leading to the appearance of the admission. These reasons are very different: the presence of slopes in the workpiece obtained by forging, stamping or casting; the absence of a clearly expressed preliminary state of the rotation element; unification of roughing and semifinishing in one operation; presence of significant interoperational unevenness of the allowance, which may exceed the allowable value for obtaining a given quality of the surface when it is processed once. For these cases, the calculation of $e_{\max }$ is carried out using the refinement coefficient [7].

Knowing the value of the diameter of the processing stage $\left(D_{i}\right)$, the value of the minimum estimated allowance $\left(z_{\min }\right)$ removing for obtaining $D_{i}$, the deviation of the previous processing stage diameter $\left(e i_{i-1}-\right.$ for the shaft, $E S_{i-1}-$ for the hole), and also taking into account that the intermediate dimensions have basic deviation $h$ (for the shaft) or $H$ (for the hole), the value of the nominal diameter of the previous machining stage $\left(D_{i-1}\right)$ for the shaft or hole, respectively, is determined according to one of the following formulas:

$$
\begin{aligned}
& D_{i-1}=D_{i}+2 z_{\min i}+\left|e i_{i-1}\right| \\
& D_{i-1}=D_{i}-2 z_{\min i}-E S_{i-1} .
\end{aligned}
$$

If for the final state of the surface, the main deviation indicated in the figure differs from $h$ or $H$, then for the outer surface of rotation in the box "Rounded size", the authors specify the maximum diameter and in the "Deviations" cell, the lower deviation, corresponding to basic deviation h. And for the inner surface of rotation in the box "Rounded size", one should specify the value of the minimum diameter, and in the "Deviation" cell one should enter the upper deviation, corresponding to main deviation $H$.

The upper and lower deviations are entered in the corresponding cell only for the state of the surface in the initial workpiece (rolling, casting, forging, stamping). In this case, according to the formula (7) or (8), the nominal diameter of the surface of revolution in the initial blank is calculated.

For all intermediate states, the nominal diameter of the surface of rotation, corresponding to its maximum (for the shaft) or the minimum (for the hole) size, is calculated by formula (7) or (8).

\section{RESULTS}

The calculated values of the diametrical dimensions, depending on the accuracy obtained by machining, or the accuracy of the method for obtaining the initial blank, should be rounded off to tenths or up to an integer in the direction of a slight increase in the allowance, for the shaft in "plus", and for the hole in "minus".

After determining the nominal diameters of intermediate stages of processing, the actual values of the minimum and maximum allowances for processing are calculated for each rotation element.
As it was mentioned before, the actual values of the minimum allowances for processing are calculated using formulas (5) or (6). In this case, the actual values of the maximum machining allowances for the shaft or hole are calculated according to formulas (9) or (10) below, respectively:

$$
\begin{gathered}
z_{\text {max } i}^{\mathrm{f}}=\left(D_{\max i-1}-D_{\min i}\right) / 2 \\
z_{\max i}^{\mathrm{f}}=\left(D_{\max i}-D_{\min i-1}\right) / 2
\end{gathered}
$$

With the correct rounding of the calculated diametrical sizes, the actual values of the minimum allowances will never be less than their calculated values. In this case, for all transitions, the actual values of the minimum allowances are greater than zero, that is, there is a guaranteed size of the allowance to ensure the required processing quality.

\section{CONCLUSION}

Comparative analysis of methods for calculating the diametrical sizes of operating blanks and allowances for processing the surfaces of rotation of axisymmetric parts allows us to draw the following conclusions.

1. An alternative method:

- does not completely contradict the theory of dimensional chains;

- allows one to abandon the experimental and installation batches when implementing the designed TP in production, since an analytically justified calculation of intermediate diametrical sizes and allowances for processing the surfaces of rotation is carried out;

- allows one to guarantee the absence of rejects in the manufacture of parts, since the calculation of maximum permissible diametrical dimensions is carried out, realizing the method of complete interchangeability.

2. When calculating the processing allowances, the calculation data for their irregularity are used, which are determined after calculating the beatings that occur during the implementation of the technology of manufacturing the axisymmetric part and checking the possibility of automatic provision without reconciliation (or with reconciliation) of the specified requirements for the mutual location (radial beats or misalignments) of the revolution surface between themselves.

3. An alternative method is suitable for use in CAD-system of TP from the point of view of the ease of development of appropriate algorithms and programs.

4. An alternative method can be used to calculate the diameters of the main holes in the body parts and allowances for processing these holes.

[1] I. Asiltürk, H. Akkuş, "Determining the Effect of Cutting Parameters on Surface Roughness in Hard Turning Using the Taguchi Method", Measurement: Journal of the International Measurement Confederation, vol. 44, issue 9, pp. 1697-1704, November 2011.

[2] I. Asiltürk, M. Çunkaş, "Modeling and prediction of surface roughness in turning operations using artificial neural network and multiple regression method", Expert Systems with Applications, vol. 38, issue 5, pp. 5826-5835, May 2011

[3] S.R. Das, A. Panda, D. Dhupal, "Analysis of surface roughness in hard turning with coated ceramic inserts: Cutting parameters effects, prediction model, cutting conditions optimization and cost analysis", Ciencia e Tecnica Vitivinicola, vol. 32, pp. 127-154, 2017. 
[4] I. Asiltürk, "Application of artificial intelligent to predict surface roughness", Experimental Techniques, vol. 38, issue 4, pp. 54-60, JulyAugust 2014.

[5] I. Asiltürk, L. Çelik, E. Canli, G. Önal, "Regression modeling of surface roughness in grinding", Advanced Materials Research, vol. 271-273, pp. 34-39, April 2011 [2011 International Conference on Advanced Materials and Information Technology Processing, AMITP 2011; Guangzhou; China, 2011].
[6] A.A. Matalin, Technology of mechanical engineering: textbook for high schools, 3rd ed., Saint Petersburg: Lan, 2010, 512 p.

[7] A.M. Dalsky, A.G. Kosilova, R.K. Meshcheryakov, A.G. Suslova. Handbook of the technologist - machine builder, 5th ed., vol. 1, Moscow: Mashinostroenie-1, 2001, 912 p.

[8] V.A. Valetov, K.P. Pompeev, Technology of instrument making, Saint Petersburg: NIU ITMO, 2013, $234 \mathrm{p}$.

[9] I.A. Ivashchenko, Technological dimensional calculations and methods of their automation, Moscow: Mechanical Engineering, 1975, 222 p. 